\title{
Estimación de masa grasa en niños chilenos: ecuaciones de pliegues subcutáneos vs densitometría de doble fotón
}

\author{
PASCUALA URREJOLA N. ${ }^{1}$, M. ISABEL HERNÁNDEZ C. ${ }^{2}$, M. GLORIA ICAZA N. ${ }^{3}$, \\ SILVIA VELANDIA A. ${ }^{1}$, M. LORETO REYES G. ${ }^{1}$, M. ISABEL HODGSON B. ${ }^{1}$ \\ 1. División de Pediatría. Unidad de Gastroenterología y Nutrición Pediátrica, Facultad de Medicina, Pontificia Universidad \\ Católica de Chile. \\ 2 Instituto de Investigación Materno Infantil. Universidad de Chile. \\ 3 Instituto de Matemática y Física, Universidad de Talca.
}

\begin{abstract}
Estimation of body fat in chilean children: comparing subcutaneous fold equations amd doble photon densitometry

Estimation of thickness of subcutabeous folds $(\mathrm{SqF})$ is a simple way to estimate body composition in children, although it has its limitations. Double photon densitometry (DXA) is considered a new standard. Objective: To compare the precision of estimation of body fat by SqF and DXA in a population of chilean children. Patients and Methods: Body fat was estimated in 128 eutrophic healthy children (70 females, 58 males), between 2 and 18 y.o. through SqF equations and DXA. Differences between both methods were evaluated through the Bland and Altman method and Lin concordance coefficient. Results: Most SqF equations had poor concordance with DXA. There was better concordance if the Westrate and Deurenberg equation was used among prepuberal and children of all ages (average difference of 2,96 $\pm 1,20 \%$ and 4,92 \pm 0,98\% and Lin concordance index of 0,50 $\pm 0,22$ and 0,43 $\pm 0,88$, respectively). Conclusions: Body fat estimated through subcutaneous fold measurements is poorly correlated with double photon densitometry among chilean children. However, until more precise equations are developed, Westrate and Deurenberg for all ages, and Westrate/Deurenberg specific for pre-puberal age can be used.

(Key words: Body composition, subcutaneous folds,percentage of body fat, double photon densitometry). Rev Chil Pediatr 2011; 82 (6): 502-511
\end{abstract}

\section{RESUMEN}

La estimación del grosor de pliegues subcutáneos (PS) es una manera simple de estimar composición corporal en niños, sin embargo, tiene limitaciones. La densitometría de doble fotón (DXA) es considerada como el nuevo estándar de referencia. Objetivo: Comparar la precisión de la estimación del porcentaje de masa grasa

Trabajo recibido el 24 de junio de 2011, devuelto para corregir el 01 de agosto de 2011, segunda versión el 08 de septiembre de 2011, aceptado para publicación el 24 de septiembre de 2011.

Apoyo Financiero: Child Health Foundation of University of Alabama.

Correspondencia a:

Dra. P Urrejola N.

E-mail.pascualaurrejola@gmail.com 
por PS con la obtenida por DXA en población infantil chilena. Pacientes y Método: Se estimó el porcentaje de masa grasa en 128 niños (70 mujeres y 58 hombres) sanos, eutróficos, entre 2 y 18 años, por diversas ecuaciones de PS y se comparó con DXA. La diferencia entre ambos mediciones fue evaluada mediante el método de Bland y Altman y el coeficiente de concordancia de Lin. Resultados: La mayoría de las ecuaciones de PS tuvieron un pobre nivel de concordancia con DXA. Sin embargo, existe una mejor concordancia entre lo calculado según PS y DXA tanto en niños pre-puberales con la fórmula específica de Westrate y Deurenberg y en niños de todas las edades, con la ecuación general de Westrate y Deurenberg (diferencia promedio de 2,96 $\pm 1,20 \%$ y $4,92 \pm 0,98 \%$ e índice de concordancia de Lin de 0,50 $\pm 0,22$ y 0,43 \pm 0,88, respectivamente) Conclusiones: La estimación del porcentaje de masa grasa a partir de los PS, tiene una baja concordancia, sin embargo, mientras no se desarrollen ecuaciones con una precisión más alta se podrían utilizar las ecuaciones de Westrate y Deurenberg a todas las edades y la de Westrate y Deurenberg específica para la edad pre-puberal. (Palabras clave: Composición corporal, pliegues subcutáneos, porcentaje de grasa corporal, densitometría de doble fotón).

Rev Chil Pediatr 2011; 82 (6): 502-511

\section{Introducción}

La determinación de la composición corporal (CC) es una herramienta útil para la evaluación del estado nutritivo a distintas edades y permite entender de manera más exacta las posibles asociaciones entre la proporcionalidad de los distintos compartimentos orgánicos y su alteración debido a diversas enfermedades $^{1,2}$. En pediatría la evaluación nutricional ha cobrado mayor importancia en el último tiempo debido al aumento en la prevalencia de enfermedades que producen variaciones en los distintos compartimentos corporales, como la obesidad, los trastornos de la conducta alimentaria, algunas enfermedades crónicas y el uso de medicamentos por períodos prolongados ${ }^{3-5}$, que inciden directamente en la CC.

La CC varía acorde de la edad, sexo y etnia del sujeto ${ }^{6,7}$, siendo el contenido de grasa corporal (GC) el componente más variable, ya que puede diferir entre individuos del mismo sexo, talla y peso ${ }^{8}$. En relación a la GC, ésta varía a medida que el sujeto crece, según el estadio de Tanner y es dependiente del origen étnico ${ }^{6,10}$. Por otro lado, durante el crecimiento y desarrollo, existe una "inmadurez química" de masa magra, dado que ésta tiene un menor contenido de potasio, menor densidad y mineralización, y mayor contenido de agua que en adultos ${ }^{6-8}$. Por esto en niños y adolescentes la CC no es constante y, por lo tanto, para su evaluación es necesario usar constantes espe- cíficas para la edad y sexo ${ }^{8-10}$. Todo esto ha significado que aún no se cuente con un método estándar y preciso para determinar CC, de uso internacional y aplicable para todas las edades, tanto en la práctica clínica, como en estudios epidemiológicos.

El desafío es desarrollar técnicas no invasivas para estimar la CC en niños, que además tengan exactitud y sean rápidas y fáciles de usar. La impedanciometría biolectrica (IBE) se basa en que la conducción de una corriente eléctrica es a través del compartimento acuoso, permitiendo estimar la masa magra y grasa a través de ecuaciones. Tiene ventajas por su simplicidad y rapidez sin embargo, dado que la CC varía en pediatría las ecuaciones deben ser validadas en diferente poblaciones ${ }^{9}$. Por otro lado, la estimación del grosor de los pliegues subcutáneos (PS) ha sido más frecuentemente utilizada ${ }^{9,15}$. Dichas mediciones tienen algunas limitaciones que incluyen: variabilidad entre examinadores, cambios en la densidad corporal con la edad y la escasa relación entre grasa subcutánea y visceral ${ }^{15}$. Esto hace que el uso de PS para estimar grasa corporal sea altamente dependiente del examinador y de la población evaluada ${ }^{13-15}$. Sin embargo, cuando las mediciones son realizadas por un examinador entrenado y se usa una ecuación población específica, el error de la determinación de la grasa corporal no debiera superar el 5\% ${ }^{7,9}$.

Un método de estudio de CC más reciente es la densitometría de doble fotón (DXA), la 
que es considerada por muchos investigadores, como el nuevo estándar de referencia ${ }^{16,17}$. La DXA es un método simple, rápido, con una duración máxima de 20 minutos, de gran aceptabilidad en los niños y sin error del examinador $^{16,17}$. La DXA basa sus mediciones en el hecho que cada componente del organismo tiene diferente densidad, la que se mide de acuerdo a un modelo multicompartamental que da información de los tres componentes del cuerpo: masa grasa, tejidos blandos libres de grasa y contenido mineral, los dos últimos son combinados y corresponden a la masa libre de grasa. La DXA es bien tolerada en la población pediátrica, sin embargo, en Chile, a pesar de estar ampliamente disponible, existen pocos centros que dispongan de software pediátrico.

El objetivo del presente estudio es comparar la precisión de la estimación de grasa corporal por PS (a través de diferentes fórmulas disponibles en la literatura), con la medición obtenida por DXA, y analizar la concordancia entre ambos métodos en población infantil chilena.

\section{Pacientes y Método}

\section{Sujetos}

Se estudiaron 128 niños entre 2 y 18 años, 70 (55\%) mujeres, provenientes de Santiago. Criterios de inclusión: antecedentes de ser recién nacido de término, con peso de nacimiento mayor a $2500 \mathrm{~g}$, sano, tener los dos apellidos chilenos, índice peso talla (IPT) entre 90 y 110\%, índice de masa corporal (IMC) entre percentil 10 y 85 de las curvas del CDC $^{18}$ y talla entre percentil 25-75. Los padres o guardianes legales firmaron el consentimiento informado aprobado por el Comité de Ética de la Pontificia Universidad Católica de Chile.

\section{Mediciones}

A cada niño se le realizó: Anamnesis para determinar patologías previas. Examen físico completo que incluyó determinación de desarrollo puberal según estadio de Tanner. Antropometría: Previo ayuno de 10 horas los sujetos fueron pesados en pesa marca SECA, calibrada (100 g de precisión) con graduación de 0,1 kg, con ropa interior y descalzos. Se realizó medición de estatura en estadiómetro incorporado a la pesa, con graduación de $0,1 \mathrm{~cm}$. El IMC fue calculado como peso/talla ${ }^{2}$. La medición de pliegues cutáneos se efectuó con un caliper Lange ${ }^{\mathrm{R}}$, en el lado izquierdo del cuerpo, en cuatro sitios: tríceps, bíceps, subescapular y suprailíaco de acuerdo al procedimiento estandarizado $^{7}$. Se usó la ecuación de Slaughter ${ }^{19}$ con los pliegues tricipital y subescapular y la ecuación de Westrate y Deurenberg ${ }^{20}$ con la utilización de 4 pliegues cutáneos, para determinar el porcentaje de masa grasa. Dichas ecuaciones (tabla 1), han sido desarrolladas en población infantil caucásica y son las más frecuentemente utilizadas en la literatura.

Densitometría de doble fotón: se realizó densitometría ósea de cuerpo total para obtener CC y \%GC, en densitómetro DXA Lunar, modelo DPX-L, Radiation Corp., Madison WI, EEUU, programa de análisis de datos versión 4.7, en el laboratorio de densitometría de la Pontificia Universidad Católica de Chile. Los valores de composición corporal fueron calculados por el instrumento.

\section{Análisis estadístico}

Los sujetos fueron reclutados por mostreo consecutivo y fueron distribuidos homogéneamente por estadios de Tanner (pre puber vs púberes) y sexo (test de exacto de Fisher y prueba t de Student, $\mathrm{p}>0,5$ respectivamente). Se utilizó estadística descriptiva para las diferentes mediciones. La relación entre el \%GC por DXA y PS fue analizada mediante Prueba t Student para muestras relacionadas. Las diferencias entre promedios de hombres y mujeres se analizaron mediante la Prueba t de Student para muestras independientes. La concordancia entre DXA y las ecuaciones de PS se evaluó mediante el método de Bland y Altman ${ }^{21}$ y el coeficiente de concordancia de $\mathrm{Lin}^{22}$. El método de Bland y Altman ${ }^{21}$ es gráfico y analítico, permitiendo discriminar cuánto difiere un nuevo método de otro ya establecido y considera que, al no conocerse el valor verdadero de los métodos estudiados, la mejor estimación es el promedio de las mediciones. La magnitud del promedio de las diferencias indica el sesgo entre las dos mediciones. 


\section{Resultados}

La edad, el desarrollo puberal, el puntaje Z de IMC y talla, y el \%GC estimado por DXA y por las ecuaciones de PS en hombres y mujeres se muestran en tablas 2 y 3 . El \% GC promedio estimado por DXA en la muestra total de hombres fue de $17,13 \pm 6,59 \%$ y de $22,71 \pm$ $7,51 \%$ en las mujeres, existiendo una diferencia estadísticamente significativa (t-student, $\mathrm{p}$ $<0,001)$ entre ambos sexos. Sin embargo, el \%GC en hombres y mujeres pre- púberes es comparable, $16,97 \pm 5,28 \%$ y $18,32 \pm 4,84 \%$, respectivamente (t-student, $\mathrm{p}>0,05$ ). Se encontraron diferencias estadísticamente signi- ficativas entre el \% GC determinada por las diferentes ecuaciones de PS en ambos sexos y en los diferentes grupos de desarrollo puberal (t-student, $\mathrm{p}<0,001$ ) (tabla 3).

Al aplicar el criterio de Bland y Altman ${ }^{21}$ para la muestra total se observó que el nivel de concordancia entre el \%GC medido por DXA y el estimado por las ecuaciones de PS fue bajo. Esto es demostrado gráficamente en las figuras 1 y 2 donde el tamaño de la diferencia promedio (DXA-PS) y la dispersión entre las mediciones es muy amplio, 5,49 $\pm 0,82 \%$ para fórmula de Slaughter ${ }^{19}$ y de 4,92 $\pm 0,98 \%$ para la de Westrate y Deurenberg ${ }^{20}$, con un 95\% límite de concordancia de 9,13 y 10,82

Tabla 1. Ecuaciones utilizadas para calcular Porcentaje de Grasa Corporal (\%GC) desde Pliegues Subcutáneos

\begin{tabular}{|c|c|c|}
\hline Autor & Población & Ecuaciones \\
\hline \multirow[t]{3}{*}{ Slaughter (19) } & $\begin{array}{l}\text { Mujeres: } \\
\text { Pre-púberes y púberes }\end{array}$ & $\begin{array}{l}\% \mathrm{GC}=1,33 \text { (tricipital }+ \text { subescapular) }-0,013 \text { (tricipital + subescapu- } \\
(\mathrm{lar})^{2}-2,5\end{array}$ \\
\hline & $\begin{array}{l}\text { Hombres: } \\
\text { Pre-púberes }\end{array}$ & $\begin{array}{l}\% \mathrm{GC}=1,21 \text { (tricipital }+ \text { subescapular) }-0,008 \text { (tricipital + subescapu- } \\
\text { lar) })^{2}-1,7\end{array}$ \\
\hline & Púberes & $\begin{array}{l}\% G C=1,21 \text { (tricipital + subescapular) }-0,008 \text { (tricipital + subescapu- } \\
(\text { ar) })^{2}-3,4\end{array}$ \\
\hline \multirow[t]{2}{*}{ Westrate y Deurenberg (20) } & $\begin{array}{l}\text { Hombres: } \\
2 \text { a } 18 \text { años: } \\
\text { Pre-púberes } \\
\text { Púberes }\end{array}$ & $\begin{array}{l}\% G C=[562-4,2(\text { edad }-2)] \text { \& }-[525-4,7(\text { edad }-2)] \\
D=1,1133-0,0561(\log \text { sumatoria } 4 \text { pliegues })+1,7\left(\text { edad } 10^{-3}\right) \\
D=1,0555-0,0352(\log \text { sumatoria } 4 \text { pliegues })+3,8\left(\text { edad } 10^{-3}\right)\end{array}$ \\
\hline & $\begin{array}{l}\text { Mujeres: } \\
2 \text { a } 10 \text { años: } \\
10 \text { a } 18 \text { años } \\
\text { Pre -púberes } \\
\text { Púberes }\end{array}$ & $\begin{array}{l}\% G C=[562-1,1(\text { edad }-2)] \propto D-[525-1,4(\text { edad }-2)] \\
\% G C=[533-7,3(\text { edad }-10) / D]-[514-8(\text { edad-10) }] \\
D=1,1187-0,063(\log \text { sumatoria } 4 \text { pliegues })+1,9\left(\text { edad } 10^{-3}\right) \\
D=1,1074-0,0504(\text { log sumatoria } 4 \text { pliegues })+1,6\left(\text { edad } 10^{-3}\right)\end{array}$ \\
\hline
\end{tabular}

D: Densidad. Sumatoria 4 pliegues: bicipital + tricipital + subescapular + suprailiaco (mm). Edad (años).

Tabla 2. Edad, peso, talla y estado puberal de los niños evaluados

\begin{tabular}{|lccc|}
\hline & $\begin{array}{c}\text { Mujeres } \\
(\mathbf{n}=\mathbf{7 0})\end{array}$ & $\begin{array}{c}\text { Hombres } \\
(\mathbf{n}=\mathbf{5 8})\end{array}$ & $\begin{array}{c}\text { Total } \\
(\mathbf{n}=\mathbf{1 2 8})\end{array}$ \\
\hline Edad (años) & $9,2 \pm 4,6$ & $8,9 \pm 6,6$ & $9,5 \pm 4,3$ \\
\hline Estadio Tanner I (n) & 39 & 36 & 75 \\
\hline Estadio Tanner II-V (n) & 31 & 22 & 53 \\
\hline Peso (kg) & $32,2 \pm 14,3$ & $34,9 \pm 15,1$ & $33,2 \pm 14,5$ \\
\hline Talla (m) & $1,30 \pm 0,23$ & $1,37 \pm 0,29$ & $1,33 \pm 0,22$ \\
\hline Puntaje Z Talla & $-0,15 \pm 0,58$ & $0,65 \pm 1,00$ & $0,25 \pm 0,89$ \\
\hline IMC (kg/m $)$ & $17,80 \pm 2,26$ & $17,67 \pm 2,06$ & $17,74 \pm 2,16$ \\
\hline Puntaje Z IMC & $0,24 \pm 0,49$ & $0,31 \pm 0,57$ & $0,16 \pm 0,78$ \\
\hline
\end{tabular}


respectivamente (tabla 4). Al aplicar el índice de concordancia de $\operatorname{Lin}^{22}$ para la fórmula de Slaughter ${ }^{19}$, éste fue débil (rc 0,55 $\pm 0,09$ ), como también para la fórmula de Westrate y Deurenberg $^{20}$ (rc 0,43 $\pm 0,88$ ) (tabla 4).

En relación al análisis según sexo ambas ecuaciones de PS fueron poco concordantes con el \%GC estimado por DXA (tabla 4 y fi- guras 1 y 2), siendo la ecuación de Westrate y Deurenberg ${ }^{20}$ en el grupo total de hombres, la con una menor diferencia promedio $(2,86 \pm$ $1,24 \%)$ y un índice de concordancia de Lin de $0,55 \pm 0,14$. Esta última ecuación en el grupo total de mujeres mostró una diferencia promedio de 6,62 $\pm 1,38 \%$ y un índice de $\operatorname{Lin}^{22}$ de 0,3 $\pm 0,11$ (tabla 4 y figuras 1 y 2 ).

Tabla 3. Comparación del Porcentaje de Grasa Corporal (\%GC) estimado por DXA y ecuaciones de Pliegues Subcutáneos

\begin{tabular}{|c|c|c|c|}
\hline & $\begin{array}{c}\text { DXA } \\
\% \text { GC } \pm 1 \text { DE }\end{array}$ & $\begin{array}{l}\text { Slaughter } \\
\% \text { GC } \pm 1 \text { DE }\end{array}$ & $\begin{array}{c}\text { Westrate y Deurenberg } \\
\% \text { GC } \pm 1 \mathrm{DE}\end{array}$ \\
\hline Total $(n=128)$ & $20,19 \pm 7,61$ & $14,64 \pm 5,58^{1}$ & $15,12 \pm 4,02^{1}$ \\
\hline Hombres $(n=58)$ & $17,13 \pm 6,59$ & $12,66 \pm 4,56$ & $14,27 \pm 4,00^{1}$ \\
\hline Hombres pre-púberes $(n=36)$ & $16,97 \pm 5,28$ & $12,47 \pm 4,10^{1}$ & $14,17 \pm 4,10^{1}$ \\
\hline Hombres púberes $(n=22)$ & $17,40 \pm 8,45$ & $12,97 \pm 5,31^{1}$ & $14,44 \pm 4,2^{1}$ \\
\hline Mujeres $(n=70)$ & $22,71 \pm 7,51$ & $16,29 \pm 5,85^{1}$ & $15,95 \pm 3,90^{1}$ \\
\hline Mujeres pre- púberes $(n=39)$ & $18,32 \pm 4,84$ & $12,64 \pm 3,69^{1}$ & $14,32 \pm 3,60^{1}$ \\
\hline Mujeres púberes $(n=31)$ & $28,25 \pm 6,57$ & $20,85 \pm 4,74{ }^{1}$ & $17,68 \pm 3,47^{1}$ \\
\hline
\end{tabular}

Tabla 4. Coeficiente de Lin y diferencias promedio del Porcentaje de Grasa Corporal (\%GC) entre DXA y ecuaciones de Pliegues Subcutáneos.

\begin{tabular}{|c|c|c|c|c|c|c|}
\hline & \multicolumn{3}{|c|}{ Slaughter } & \multicolumn{3}{|c|}{ Westrate- Deurenberg } \\
\hline & $\begin{array}{c}\text { Diferencia } \\
\text { Promedio } \\
\% \text { GC } \\
\text { ( } \pm 95 \% \text { IC) }\end{array}$ & $\begin{array}{c}95 \% \\
\text { Límite de } \\
\text { concordancia }\end{array}$ & $\begin{array}{c}\text { Coeficiente de } \\
\text { concordancia } \\
\text { de Lin } \\
( \pm 95 \% \text { IC) }\end{array}$ & $\begin{array}{c}\text { Diferencia } \\
\text { Promedio } \\
\% \text { GC } \\
\text { ( } \pm 95 \% \text { IC) }\end{array}$ & $\begin{array}{c}95 \% \\
\text { Límite de } \\
\text { concordancia }\end{array}$ & $\begin{array}{c}\text { Coeficiente de } \\
\text { concordancia } \\
\text { de Lin } \\
( \pm 95 \% \text { IC })\end{array}$ \\
\hline Total & $\begin{array}{c}5,491 \\
( \pm 0,82)\end{array}$ & 9,13 & $\begin{array}{c}0,55^{2} \\
( \pm 0,09)\end{array}$ & $\begin{array}{c}4,92^{1} \\
( \pm 0,98)\end{array}$ & 10,82 & $\begin{array}{c}0,43^{2} \\
( \pm 0,88)\end{array}$ \\
\hline $\begin{array}{l}\text { Total } \\
\text { Pre-púberes }\end{array}$ & $\begin{array}{c}5,111 \\
( \pm 1,47)\end{array}$ & 7,15 & $\begin{array}{c}0,4^{2} \\
( \pm 0,12)\end{array}$ & $\begin{array}{c}3,37^{1} \\
( \pm 0,38)\end{array}$ & 7,35 & $\begin{array}{c}0,51^{2} \\
( \pm 0,13)\end{array}$ \\
\hline $\begin{array}{l}\text { Total } \\
\text { Púberes }\end{array}$ & $\begin{array}{c}6,00^{2} \\
( \pm 0,22)\end{array}$ & 11,36 & $\begin{array}{c}0,54^{2} \\
( \pm 0,14)\end{array}$ & $\begin{array}{c}7,12^{2} \\
( \pm 0,32)\end{array}$ & 13,31 & $\begin{array}{c}0,33^{2} \\
( \pm 0,12)\end{array}$ \\
\hline Hombres & $\begin{array}{c}4,47^{1} \\
( \pm 1,04)\end{array}$ & 7,76 & $\begin{array}{c}0,57^{2} \\
( \pm 1,21)\end{array}$ & $\begin{array}{c}2,86^{1} \\
( \pm 1,24)\end{array}$ & 9,28 & $\begin{array}{c}0,55^{2} \\
( \pm 0,14)\end{array}$ \\
\hline Mujeres & $\begin{array}{c}6,33^{1} \\
( \pm 1,23)\end{array}$ & 9,88 & $\begin{array}{c}0,48^{2} \\
( \pm 1,23)\end{array}$ & $\begin{array}{c}6,621 \\
( \pm 1,38)\end{array}$ & 10,02 & $\begin{array}{c}0,32^{2} \\
( \pm 0,11)\end{array}$ \\
\hline $\begin{array}{l}\text { Hombres } \\
\text { Pre-púberes }\end{array}$ & $\begin{array}{c}4,50^{1} \\
( \pm 1,17)\end{array}$ & 6,80 & $\begin{array}{c}0,50^{2} \\
( \pm 1,68)\end{array}$ & $\begin{array}{c}2,80^{1} \\
( \pm 1,23)\end{array}$ & 6,91 & $\begin{array}{c}0,60^{2} \\
( \pm 0,17)\end{array}$ \\
\hline $\begin{array}{l}\text { Hombres } \\
\text { púberes }\end{array}$ & $\begin{array}{c}4,43^{1} \\
( \pm 2,11)\end{array}$ & 9,33 & $\begin{array}{c}0,64^{2} \\
( \pm 0,18)\end{array}$ & $\begin{array}{c}2,96^{1} \\
( \pm 1,20)\end{array}$ & 12,14 & $\begin{array}{c}0,50^{2} \\
( \pm 0,22)\end{array}$ \\
\hline $\begin{array}{l}\text { Mujeres } \\
\text { Pre- púberes }\end{array}$ & $\begin{array}{c}5,68^{1} \\
( \pm 1,22)\end{array}$ & 7,38 & $\begin{array}{c}0,33^{2} \\
( \pm 0,25)\end{array}$ & $\begin{array}{l}3,89^{1} \\
( \pm 1,2)\end{array}$ & 7,68 & $\begin{array}{c}0,42^{2} \\
( \pm 0,19)\end{array}$ \\
\hline $\begin{array}{l}\text { Mujeres } \\
\text { púberes }\end{array}$ & $\begin{array}{c}7,14^{1} \\
( \pm 2,35)\end{array}$ & 12,27 & $\begin{array}{c}0,17 \text { ns } \\
( \pm 0,16)\end{array}$ & $\begin{array}{l}10,57^{2} \\
( \pm 2,1)\end{array}$ & 10,77 & $\begin{array}{c}0,11 \text { ns } \\
( \pm 0,10)\end{array}$ \\
\hline
\end{tabular}

${ }^{1} \mathrm{P}<0,05 .{ }^{2} \mathrm{P}<0,005$. ${ }^{n}$ no significativo. 


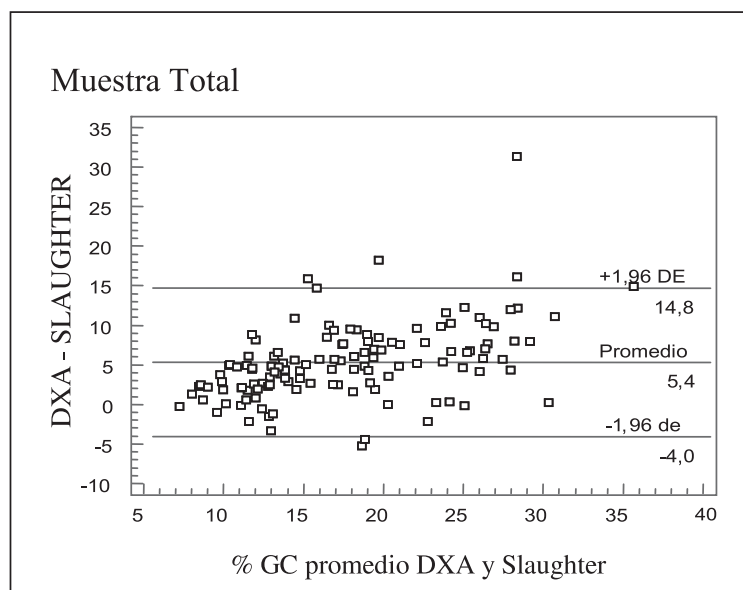

Muestra Total: Pre púber

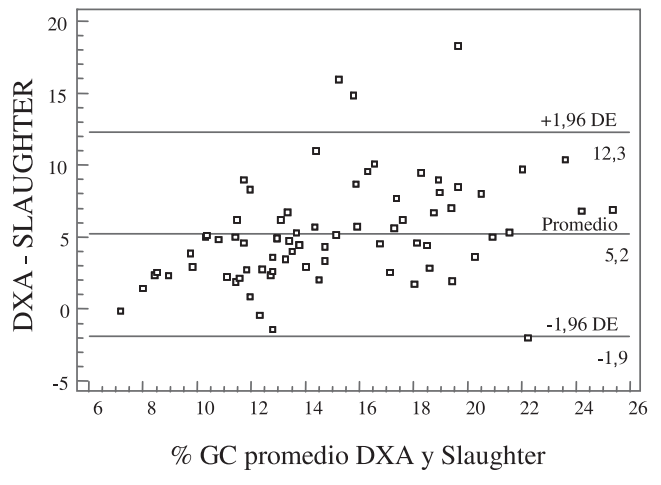

Muestra Total: Púber

\section{Hombres Total}


Mujeres Total

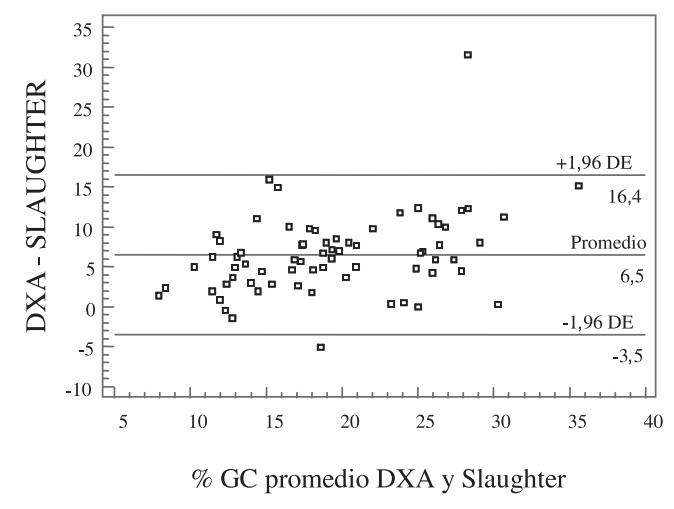

Figura 1. Comparación del Porcentaje de Grasa Corporal (\%GC) estimado por fórmula de Slaughter y DXA. Método de Bland y Altman 


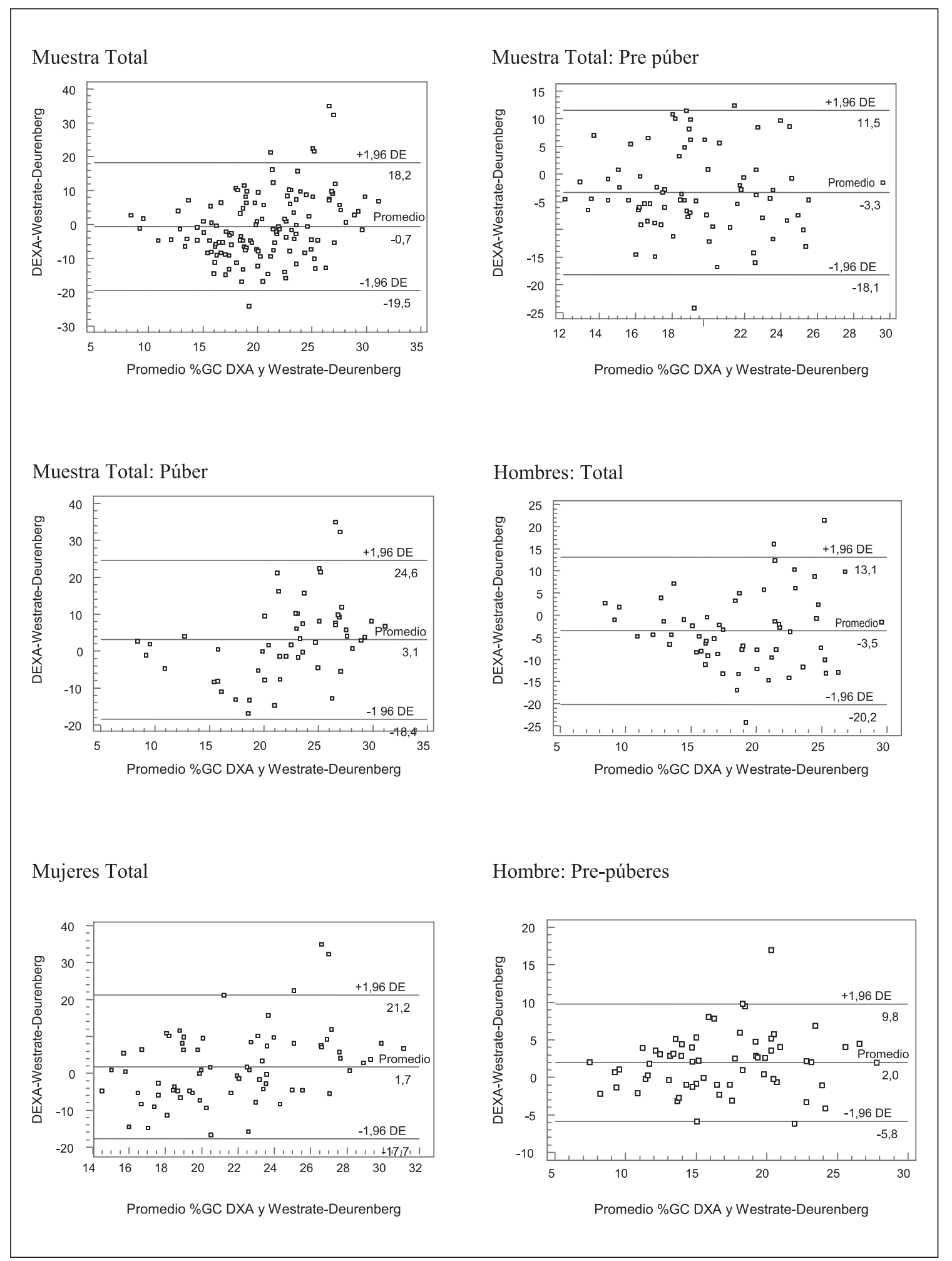

Figura 2. Comparación del Porcentaje de Grasa Corporal (\%GC) estimado por fórmula de Westrate - Deurenberg y DXA. Método de Bland y Altman 
$\mathrm{Al}$ analizar la muestra según desarrollo puberal la ecuación de Slaughter ${ }^{19}$, presentó un bajo nivel de concordancia con el \%GC estimado por DXA en pre-púberes y púberes. Esto es demostrado gráficamente en las figuras 1 y 2 donde el tamaño de la diferencia promedio y la dispersión entre las mediciones es muy amplio, 5,11 \pm 1 , 47\% para la fórmula de Slaughter ${ }^{19}$ en pre-púberes y de 6,00 $\pm 0,22 \%$ para púberes, con un 95\% límite de concordancia de 7,15 y 11,36 respectivamente (tabla 4) y un índice de concordancia de $\operatorname{Lin}^{22}$ de 0,51 \pm 0,13 y $0,33 \pm 0,12$. Sin embargo, la ecuación de Westrate y Deurenberg ${ }^{20}$ en pre-púberes mostró una diferencia promedio menor con \%GC por DXA, 3,37 $\pm 0,38 \%$, con un $95 \%$ límite de concordancia de 7,35 y coeficiente de concordancia de $\operatorname{Lin}^{22}$ de $0,51 \pm 0,13$. En púberes la ecuación de Westrate y Deurenberg ${ }^{20}$ mostró una diferencia promedio con \%GC por DXA de 7,12 \pm 0,32\%, con 95\% límite de concordancia de 13,31 y un coeficiente de concordancia de $\operatorname{Lin}^{22}$ de $0,33 \pm 0,12$ (tabla 4 y figuras 1 y 2). Al analizar la muestra según sexo y desarrollo puberal la ecuación de Westrate y Deurenberg ${ }^{20}$ para hombres pre-púberes fue la que tuvo una menor diferencia promedio $(2,80$ \pm 1,23\%,95\% límite de concordancia de 6,91 y un coeficiente de concordancia de $\operatorname{Lin}^{22}$ de 0 , $60 \pm 0,17$ (tabla 4 y figuras 1 y 2).

\section{Discusión}

Este estudio evaluó la comparabilidad de la estimación de grasa corporal usando DXA y la medición de PS por fórmulas pediátricas, en niños de 2 a 18 años, sanos y eutróficos. De las ecuaciones de PS utilizadas, tanto la ecuación de Slaughter ${ }^{19}$ como la de Westrate y Deurenberg ${ }^{22}$, tienen una baja concordancia con la medición realizada por DXA.

En la actualidad DXA es reconocida como un nuevo estándar de referencia de CC basado en diversos trabajos, tanto en animales, como en seres humanos, los que avalan su precisión y seguridad, atribuyéndole un error de 1,5-2\% en la estimación del \%GC comparado con hidrodensitometría, agua corporal total por dilución con óxido de deuterio y modelo de cuatro compartimentos $^{23,27}$.
La determinación de la GC utilizando la suma de pliegues subcutáneos (PS) se basa en los supuestos de que el grosor del tejido adiposo subcutáneo refleja una proporción constante de la GC total y que los sitios seleccionados para su medición, representan el grosor promedio del tejido subcutáneo. Las ecuaciones utilizadas en adultos para estimar CC y determinar GC desde el grosor de PS parecen ser inapropiadas en niños, ya que sobreestiman el contenido graso. En este estudio se evaluaron fórmulas validadas en población pediátrica como la ecuación de Slaughter ${ }^{19}$ y la de Westrate Deurenberg ${ }^{20}$. Estas ecuaciones toman en consideración los cambios de la relación entre el grosor de diferentes PS y las variaciones en la densidad de masa magra dependientes de la edad.

Otra variable a considerar en la validación de los PS, es el estado nutricional de los sujetos estudiados. Existe evidencia en la literatura respecto a que la medición de masa grasa a base de PS subestima la masa grasa a medida que el porcentaje de masa grasa aumenta. Las causas atribuibles a esta imprecisión serían, el aumento comparativo de la grasa subcutánea a medida que aumenta el porcentaje de masa grasa ${ }^{24-25}$ y también la influencia de las variaciones en la distribución de la grasa ${ }^{26}$ y la dificultad técnica en la obtención de los PS en personas obesas ${ }^{26}$. Para evitar los inconvenientes previos, este estudio fue realizado en población eutrófica, estado nutricional similar al que tenía la población en que fueron desarrolladas todas las ecuaciones estudiadas. Además, cuando se determinó \%GC por DXA, en la muestra total se observó que los hombres tenían un \%GC menor que las mujeres, sin embargo, el \%GC en hombres y mujeres prepúberes fue similar. Estos resultados concuerdan a lo encontrado en un estudio realizado por Sala et $\mathrm{al}^{27}$, donde analizaron composición corporal en niños canadienses utilizando DXA y los comparó con población infantil argentina y holandesa.

A pesar de lo anterior, el nivel de concordancia encontrado entre el \%GC, estimado por los PS (ecuación de Slaughter y de Westrate y Deurenberg) y DXA en la población total fue pequeño, tanto por el método de Bland y Alt- 
$\operatorname{man}^{21}$ como por el coeficiente de concordancia de $\operatorname{Lin}^{22}$. De las fórmulas analizadas, Westrate y Deurenberg ${ }^{20}$, en la población total y en niños pre-púberes, fueron las que tuvieron una mejor concordancia. Estos resultados se contraponen al trabajo de Wong et $\mathrm{l}^{28}$, quienes validaron el uso de las ecuaciones de Slaughter ${ }^{19}$ en niñas norteamericanas de 9 a 17 años, como también con otro estudio de validación realizado en hombres pre-púberes ${ }^{30}$. En un estudio reciente realizado por Rodríguez et $\mathrm{al}^{31}$, en población adolescente española, encontraron que la precisión de las ecuaciones de Westrate y Deurenberg ${ }^{20}$ y de la de Slaughter ${ }^{19}$, para evaluar \%GC en adolescentes, era pobre a nivel individual.

Tanto la ecuación de Slaughter ${ }^{19}$ como las de Westrate y Deurenberg ${ }^{20}$, fueron desarrolladas en población infantil caucásica. Numerosos estudios han demostrado que la composición corporal es dependiente del origen étnico de la persona, ya que tanto la masa magra, como el contenido de mineral óseo presentan variaciones con relación a la etnia, lo que orienta a pensar en la participación del componente genético ${ }^{9,10}$. A pesar que se supone que la población chilena tiene una CC semejante a la de origen caucásico, la falta de concordancia de las ecuaciones de Slaughter ${ }^{19}$ y de Westrate y Deurenberg ${ }^{20}$ podría estar influenciada por variantes genéticas diferentes a las de las poblaciones de las que se originaron dichas fórmulas. El estudio de Sala et al ${ }^{33}$, encontró pequeñas diferencias geográficas en la CC en los niños estudiados.

Acorde con nuestro mejor conocimiento, ésta es la primera vez que ecuaciones de PS se comparan con un método de referencia de estimación de CC como es la DXA, en un grupo grande de niños pre-púberes y púberes en población chilena infantil.

Los resultados obtenidos en este estudio permiten concluir que en niños chilenos, sanos y eutróficos, la estimación del \%GC a partir de los PS, tiene una baja concordancia con la medición realizada por DXA. Sin embargo, mientras no se desarrollen ecuaciones con una precisión más alta se podría, en poblaciones como la nuestra, utilizar las ecuaciones de Westrate y Deurenberg ${ }^{24}$ a todas las edades y la de Westrate y Deurenberg ${ }^{24}$ específica para la edad pre-puberal.

\section{Referencias}

1.- Flegal KM, Graubard BI, Williamson DF, Gail MH: Excess deaths associated with underweight, overweight, and obesity. JAMA 2005; 293 (15): 1861-7.

2.- Baumgartner RN, Heymsfield SB, Roche AF: Human body composition and the epidemiology of chronic disease. Obesity Research 1995; 3: 73-95.

3.- Freedman DS, Mei Z, Srinvasan SR, Berenson GS, Dietz $W H$ : Cardiovascular risk factors and excess adiposity among overweight children and adolescents. The Bogolusa Heart Study. J Pediatr 2007; 150: 12-7.

4.- Thibault R, Cano N, Pichard C: Quantification of lean tissue losses during cancer and HIV infection/AIDS. Curr Opin Clin Nutr Metab Care 2011; 14 (3): 261-7.

5.- Haas VK, Kohn MR, Clarke SD, et al: Body composition changes in female adolescents with anorexia nervosa. Am J Clin Nutr 2009; 89 (4): 1005-10.

6.- Fomon SJ, Haschke F, Ziegler EE, Nelson SE: Body composition and constants for children from birth to age 10 years. Am J Clin Nutr 1982; 35: 1169-75.

7.- Martin AD, Drinkwater DT: Variability in the measures of body fat. Assumptions or techniques? Sports Med 1991; 11: 277-88.

8.- Ellis KJ, Shypailo RJ, Abrams SA, Wong W: The reference child and adolescent model of body composition. Ann N Y Acad Sci 2000; 700: 375-82.

9.- Eisenmann JC. Heelen KA, Welk GJ: Assessing body composition among 3-to 8-year-old children: anthropometry, BIA, and DXA. Obes Res 2004; 12: 1633-40.

10.- Forbes GB: En: Body composition in infancy, childhood and adolescence: Human body composition. Growth, aging, nutrition and activity. ed. Spring Verlag 1987; 125-68.

11.- Dietz WH, Robinson TN: Use of body mass index (BMI) as a measure of overweight in children and adolescents. J Pediatr 1998; 132: 191-3.

12.- Horlik M: Body mass index in childhood-measuring a moving target. J Clin Endocrinol Metab 2001; 86: 405960.

13.- Lohman TG: Skinfolds and body density and their relation to body fatness: A review. Hum Biol 1981; 53: 181-225.

14.- Sutcliffe JF: A review of in vivo experimental methods to determine the composition of the human body. Phys Med Biol 1996; 41: 791-833.

15.- Durnin JV, de Bruin H, Feunekes GI: Skinfold thick- 
ness: Is there a need to be very precise in their location? Br J Nutr 1997; 77: 3-7.

16.- Goran MI Driscoll P, Johnson R, Nagy TR, Hunter $G$ : Cross-calibration of body-composition techniques against dual-energy X-ray absorptiometry in young children. Am J Clin Nutr 1996; 63: 299-305.

17.- Sopher AB, Thornton JC, Wang J, Pierson RN Jr, Heymsfield SB, Horlick M: Measurements of percentage body fat in 411 children and adolescents: A comparison of dual-energy X-ray absorptiometry with a four-compartment model. Pediatrics 2004; 113: 1285-90.

18.- Kuczmarski RJ, Ogden CL, Grummer-Strawn LM, et al: Advance data from vital and health statistics. Hyattsville: National Center for Health Statistics, 2000; 314: 1-28. Available at: http://www.cdc.goc/growthcharts

19.- Slaughter MH, Lohman TG, Boileau RA, et al: Skinfold equations for estimation of body fatness in children and youth. Hum Biol 1988; 60: 709-23.

20.- Westrate JA, Deurenberg P: Body composition in children: proposal for a method for calculating body fat percentage from total body density or skinfold-thickness measurements. Am J Clin Nutr 1989; 50: 1104-15.

21.- Bland JM, Altman DG: Statistical methods for assessing agreement between two methods of clinical measurement. Lancet 1986; 8: 307-10.

22.- Lin L: A concordance correlation coefficient to evaluate reproducibility. Biometrics 1989; 45: 255-68.

23.- Pintauro S, Nagy TR, Duthie C, Goran MI: Crosscalibration of fat and lean measurements by dual energy X-ray absorptiomtry to pig carcass analysis in the paediatric body weight range. Am J Clin Nutr 1996; 63: 293-9.

24.- Deurenberg P, Pieters J, Hautvast J: The assessment of the body fat percentage by skinfold thickness measurements in childhood and young adolescence. Br J Nutr 1990; 63: 293-303.

25.- Westrate JA, Deurenberg P, Tinteren H: Indices of body fat distribution and adiposity in Dutch children from birth to 18 years of age. Int J Obes 1989; 13: 465-77.

26.- Gray D, Bray G, Bauer M, et al: Skinfold thickness measurements in obese subjects. Am J Clin Nutr 1990; 51: 571-7.

27.- Sala A, Webber CE, Morrison J, Beaumont LF, Barr $R D$ : Whole-Body Bone Mineral content, Lean Body Mass, and Fat mass Measured by Dual-Energy X-Ray Absoptiometry in a Population of Normal Canadian Children and Adolescents. CARJ 2007; 58: 46-52.

28.- Wong WW, Stuff JE, Butter NF, Smith EO, Ellis KJ: Estimating body fat in African American and white adolescent girls: a comparison of skinfold-thickness equations with a 4-compartment criterion model. Am J Clin Nutr 2000; 73: 348-54.

29.- Reilly JJ, Wilson J, Durnim JVGA: Determination of body composition from skinfold thickness: a validation study. Arch Dis Child 1995; 73: 305-10.

30.- Rodríguez G, Moreno LA, Blay MG, Fleta VA Sarria A, Bueno M: Body fat measurement in adolescents: comparison of skinfold thickness equations with DualEnergy X Ray Absorptiometry. Eur J Clin Nutr 2005; 59: 1158-66. 